\title{
Targeted next-generation sequencing study in familial ALS-FTD Portuguese patients negative for C9orf72 HRE
}

\author{
Marta Gromicho ${ }^{1}$ (D) Ana Margarida Coutinho ${ }^{2} \cdot$ Ana Catarina Pronto-Laborinho $^{1} \cdot$ Rita Raposeiro $^{2}$. Joana Tavares ${ }^{2}$. \\ Diana Antunes ${ }^{2,3} \cdot$ Mamede de Carvalho ${ }^{1,4}$
}

Received: 8 May 2020 / Revised: 25 June 2020 / Accepted: 30 June 2020 / Published online: 7 July 2020

(c) Springer-Verlag GmbH Germany, part of Springer Nature 2020

\begin{abstract}
Amyotrophic lateral sclerosis (ALS) is a neurodegenerative disease with clinical and etiological heterogeneity and a complex genetic contribution. Clinical, neuropathological, and genetic evidence revealed that ALS and frontotemporal dementia (FTD) are in part of a single disease continuum. Genetic causes have been identified in sporadic (SALS) and familial patients (FALS) and the recurrent genetic factor underlying ALS and FTD is the C9orf72 hexanucleotide repeat expansion (HRE). However, in our population, the concomitance of ALS and FTD cannot be explained by C9orf72 HRE in many FALS and SALS cases. Our aim is to further understand the genetic basis of ALS in Portuguese patients. 34 patients with FALS or SALS-FTD, negative for $C 9$ orf $72 \mathrm{HRE}$, were screened for rare variants in a panel of 29 relevant genes by next-generation sequencing. We detected 15 variants in 11 genes, one classified as pathogenic in TARDBP, two as likely pathogenic in $T A R D B P$ and $P R P H$, and the others as variants of unknown significance (VUS). Gene variants, including VUS, were found in $41.2 \%$ FALS patients and 40\% SALS-FTD. In most patients, no potential pathogenic variants were found. Our results emphasize the need to enhance the efforts to unravel the genetic architecture of ALS-FTD.
\end{abstract}

Keywords Amyotrophic lateral sclerosis · Frontotemporal dementia $\cdot$ Next-generation sequencing $\cdot$ Genetic screening . Gene variants

\section{Introduction}

Amyotrophic lateral sclerosis (ALS) is an adult onset, progressive neurodegenerative disease, characterized by the loss of both upper motor neurons (UMN) and lower motor neurons (LMN) at spinal cord and bulbar levels. Additionally, cognitive and/or behavioural impairment due to the

Marta Gromicho

martalgms@gmail.com

1 Instituto de Medicina Molecular and Institute of Physiology, Faculdade de Medicina da Universidade de Lisboa, Av. Professor Egas Moniz, 1648-028 Lisbon, Portugal

2 GenoMed-Diagnósticos de Medicina Molecular SA, Instituto de Medicina Molecular, Faculdade de Medicina da Universidade de Lisboa, Lisbon, Portugal

3 Medical Genetics Department, Hospital de Dona Estefânia, Centro Hospitalar Lisboa Central, Lisbon, Portugal

4 Department of Neurosciences and Mental Health, Hospital de Santa Maria, Centro Hospitalar Universitário de Lisboa Norte, Lisbon, Portugal involvement of prefrontal cortex is detected in $30-50 \%$ of ALS patients, and $13 \%$ of patients fulfil the clinical diagnostic criteria for frontotemporal dementia (FTD) [1]. Conversely, up to $50 \%$ of FTD patients develop signs of motor involvement with about $15 \%$ meeting the diagnostic criteria of ALS [2]. Thus, based on clinical, neuropathological, and genetic links, ALS and FTD are now recognized to be extremes in the phenotypic spectrum of a single disease: the ALS-FTD continuum [1].

Although most ALS patients have sporadic disease, in which no family history can be discernible (SALS), the population-based rate of familial ALS (FALS) is about 20\% $[3,4]$. In our ALS population, FALS was disclosed in $11.6 \%$ of patients [5].

Population-based frequency of the mutation rate in ALS European populations estimates that variants in four genes account for $51 \%$ of FALS and $6.2 \%$ of SALS, being the most common the $C 9$ orf 72 hexanucleotide repeat expansion (FALS 39.6\%, SALS 4.6\%), followed by SOD1 (FALS $6.9 \%$, SALS $0.8 \%$ ), TARDBP (FALS $2.7 \%$, SALS $0.6 \%$ ), and FUS mutations (FALS 1.8\%, SALS 0.2\%) [6]. In FTD, 
the frequency of the C9orf72 expansions in Western Europe was about $10 \%$, with $18.5 \%$ in familial and $6.3 \%$ in sporadic FTD patients [7]. Moreover, the C9orf72 HRE accounts for up to $88 \%$ of familial ALS-FTD patient series and is thus the major genetic cause of the FTD-ALS spectrum [8-11]. In addition to $C 9$ orf72, other genes in ALS/FTD spectrum include: CCFN, CHCHD10, CHMP2B, OPTN, SQSTM1, $T B K 1, U B Q L N 2$, and $V C P[12,13]$.

A large population-based pedigree study confirms that up to $50 \%$ of variance in ALS has a genetic basis and estimate the overall mean lifetime heritability of ALS in C9orf72negative patients to be $36.9 \%$ (95\%CI, 19.8-53.9\%) [14]. More than 30 genes have been described to confer a higher risk for ALS [15-17] and their contribution is complex. ALS has a monogenic component of rare high-penetrant variants, an oligogenic component of rare intermediate penetrant variants, and a multifactorial component of common risk variants, possibly all under the influence of gene-environment interactions [18]. A multistep process has been hypothesized as necessary for the onset of the disease $[19,20]$ and the number of steps required is reduced in patients with genetic variants [21].

We have previously found that, in the Portuguese population, C9orf72 HRE accounts for $4.6 \%$ of sporadic ALS and $37.5 \%$ of FALS, while SOD1 mutations are rare $(0.83 \%)$. As expected, patients with C9orf72 HRE have a higher prevalence of FTD however, but the concomitance of ALS and FTD cannot be explained by C9orf72 HRE in $42 \%$ of FALS and $83 \%$ of sporadic cases. Also, two ALS patients with FTD onset were C9orf72 HRE negative. Not only a positive family history for ALS or FTD, but also the presence of comorbid FTD increases the chance of having a genetic variant by 3.5 [22]. Thus, genetic screening is indicated in patients manifesting both diseases without the C9orf 72 HRE, regardless of family history of disease [23, 24].

Thus, whenever possible, our patients with familial ALS and/or FTD negative for $C 9$ orf $72 \mathrm{HRE}$ were investigated for rare variants in a panel of ALS genes by next-generation sequencing (NGS). Our aim is to further understand the genetic basis of ALS in Portuguese patients.

\section{Methodology}

\section{Patients}

Patients were recruited by MdC in the Neuromuscular Unit, Department of Neurosciences, Hospital de Santa MariaCHULN, the main ALS center in Portugal. ALS Patients met the revised El-Escorial criteria [25], including eligible patients with PMA (progressive muscular atrophy) and PLS (primary lateral sclerosis). Diagnosis was supported by neurophysiological evaluation [26]. All included patients had disease progression.

Clinical information was recorded following a structured assessment tool, incorporating neurological examination [27]. FALS was defined based on familial history, and was classified as definitive, probable, or possible according to the criteria of Byrne and co-workers [28].

The presence of comorbid FTD on clinical judgment (always by the same clinician, $\mathrm{MdC}$ ) was supported by the International consensus criteria for possible behavioural FTD, i.e., presence of at least three of six clinically discriminating features (disinhibition, apathy/inertia, loss of sympathy/empathy, perseverative/compulsive behaviours, hyperorality, and dysexecutive neuropsychological profile) [29]. The presence of language dysfunction was clinically appreciated and selected cases were evaluated by ECAS (Edinburgh Cognitive and Behavioural ALS Screen) to confirm clinical diagnosis.

The functional rate of decay $(\Delta \mathrm{FS})$ was calculated as follows: (48-total ALSFRS-R at diagnosis)/duration in months from weakness onset to diagnosis. We considered the time since motor onset, because some patients had previous FTD symptoms and the Revised ALS Functional Rating Scale (ALSFRS-R) only evaluates motor functional impairment [30].

All patients that agreed to be tested and that signed inform consent were tested for C9orf72 HRE in GenoMed-Diagnósticos de Medicina Molecular, S.A, as previously described [31]. We excluded those with marked signs of respiratory insufficiency, and who were psychologically stressed or had major depression. From a total of 282 patients tested, 265 (94\%) were negative for C9orf72 HRE (less than $30 \mathrm{GGG}$ GCC hexanucleotide repeats in the first intron of $C 9$ orf 72 gene).

In the group of 265 C9orf72-negative patients, there were 19 unrelated FALS patients ( $7.2 \%$ of the total), of which six had concomitant FTD, and 23 SALS with concomitant FTD ( $8.7 \%$ of the total). From this group, enough DNA quantity and quality for further genetic testing with Next-Generation Sequencing were available from 32 patients (Fig. 1). Additionally, two family members with ALS of two FALS patients (a brother and a nephew) were also sequenced. All patients were Caucasians of European origin (Portugal), except for one with African origin.

\section{Targeted gene enrichment and sequencing}

Genomic DNA was isolated from whole blood samples using a standard in-house salting-out method. Wholeexome libraries were prepared using the SureSelect Human All Exon v6 kit (Agilent). Target regions were sequenced (paired-end) on an Illumina platform (NovaSeq 6000) with 150 base read length, with a medium read depth of at least 


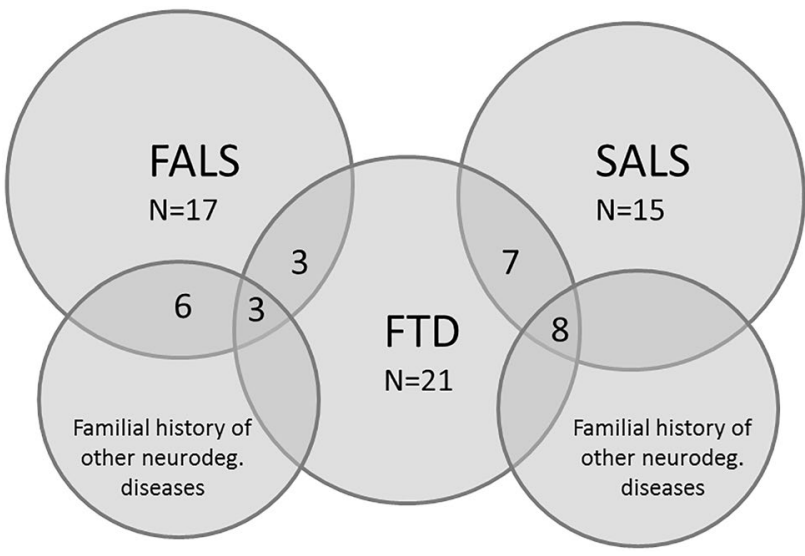

Fig. 1 Schematic diagram with the categorization of the studied patients; FALS familial amyotrophic lateral sclerosis, $S A L S$ sporadic amyotrophic lateral sclerosis, FTD frontotemporal dementia, other neurodegenerative disorders include Alzheimer disease, Parkinson disease, and FTD and unspecified dementia

60x. The exonic and splice-site DNA sequence regions of 29 ALS relevant genes were analysed in 34 patients, all negative for C9orf72: $A N G, A T X N 2, C C N F, C H C H D 10$, CHMP2B, DCTN1, ERBB4, FIG4, FUS, hnRNPA1, hnRNPA2B1, KIF5A, MATR3, NEFH, OPTN, PFN1, PRPH, SIGMAR1, SOD1, SQSTM1, SPG11, TARDBP, TBK1, TREM2, TRPM7, TUBA4A, UBQLN2, VAPB, and VCP.

In four patients, the following additional genes were studied: ALS2, ANXA11, ATP13A2, BSCL2, CHCHD2, DAO, ELP3, ERLIN1, EWSR1, GLE1, GLT8D1, GNE, GRN, HSBP1, MAPT, NEK1, PLEKHG5, SETX, SLC52A2, SLC52A3, TAF15, TIA1, TRPV4, UNC13A, and VRK1. No variants were detected in none of them.

The coverage of the gene panel was higher than $98 \%$ for variants with a read depth of $>20 X$. Thus, only those variants with insufficient quality or coverage are confirmed by Sanger sequencing. Genetic analysis was performed at GenoMed—Diagnósticos de Medicina Molecular, S.A.

\section{Bioinformatic data analysis}

Raw sequencing data in fastq format were assessed for quality with fastp software (version 0.19.8) and aligned with BWA (version 0.7.17) against the human reference genome GRCh37.

For variant calling, we used GATK-HaplotypeCaller [32] (version 4.1.1.0), FreeBayes [33] (version 1.1.0.46), and SAMtools mpileup [34] (version 1.9). While the first two apply a haplotype-based approach, the latter use an alignment-based approach. By taking advantage of both approaches, variants located in coding regions, including the splice site, called by at least two of the callers with a read depth of 20X or more were selected for further analysis.
Variants with minor allele frequency $(\mathrm{MAF})>0.01$ were filtered out. Reported variants are below the highest diseasespecific maximum credible population allele frequency (AF) for which the observed allele count is compatible with pathogenicity [35]. AF in control populations and ALS cases was determined using variant frequencies from The Genome Aggregation Database (gnomAD, version 2.1.1) and from project MinE data browser (https://databrowser.projectmin e.com/) which currently contains WGS data from 4366 ALS cases and 1832 controls [36]. Variants were also searched at the ALS Data Browser v3, ALSdb, New York City, New York (URL: https://alsdb.org) [date $(03,2020)$ accessed], a catalogue of genetic variants identified from $3317 \mathrm{Cau}-$ casian ALS patients [37]. The clinical impact of coding variants was annotated with the National Center for Biotechnology Information (NCBI) ClinVar database (https:// www.ncbi.nlm.nih.gov/clinvar/) and classified according to the American College of Medical Genetics and Genomics (ACMG) guidelines [38]. Therefore, synonymous variants were reported if computational evidence suggested a possible impact on splicing [38]. To assess the potential functional consequences of detected variants, we used wellestablished computational methods based on evolutionary conservation, protein structure, and/or sequence homology: MutationTaster [39], PolyPhen-2 HVAR [40], and SIFT [41], that predict the effect of the change on protein function; the ensemble methods, Combined Annotation Dependent Depletion (CADD), Rare Exome Variant Ensemble Learner (REVEL) [42], MutationAssessor [43], Meta LR [44], as well as conservation scores as computed with the Genomic Evolutionary Rate Profiling (GERP) [45]. Thus, the Variant Effect Predictor tool (Ensembl.org Release 99) [46] and the tools available from the dbNSFP database v3.0 (MetaSVM, MetaLR, PROVEAN, SIFT, Polyphen2, MutationTaster, MutationAssessor) [47], and LoFtool [48] were used to predict the effect of each variant on the corresponding protein. The probabilities of a variant being splice altering were predicted with two ensemble learning methods, adaptive boosting (AdaBoost, ada score) and random forests (rf score) [49].

\section{Results}

Thirty-two unrelated patients, plus two family members, totalizing 34 patients (2 PLS, 7 PMA and 25 ALS) negative for the presence of C9orf72 HRE and with a positive family history for ALS and/or with concomitant FTD were analysed with targeted NGS. Thus, considering only unrelated patients, $53 \%$ were classified as FALS (17/32). The studied population consisted of 14 males and 20 females with an average age of disease onset of $65.9 \pm 13.4$ years. Fourteen patients had spinal-onset, nine had bulbar-onset, one had respiratory-onset, two had a generalized-onset, 
and 8 had FTD-onset. Besides, 13 other patients developed FTD during disease progression. They all presented the behavioural phenotype of FTD, but in a few language changes were associated at onset; in most of them, language dysfunction was clear over disease progression, as identified by abnormalities in writing (agrammatism and limited vocabulary). In the patients with milder cognitive changes at onset, ECAS confirmed abnormal results, and in all patients, cognition deteriorated over disease progression. Eighteen patients had predominant LMN signs, 15 had predominant UMN signs, and in one, there was no UMN vs. LMN predominance.

The targeted NGS data showed pathogenic variants, likely pathogenic variants and those of uncertain significance (VUS) in the following 11 genes: CCNF (Cyclin F), CHMP2B (Charged Multivesicular Body Protein 2B), ERBB4 (Erb-B2 Receptor Tyrosine Kinase 4), FUS (FUS RNA Binding Protein), NEFH (Neurofilament Heavy), $P R P H$ (Peripherin), SPG11 (Vesicle Trafficking Associated, Spatacsin), TARDBP (TAR DNA Binding Protein), TBKI (TANK Binding Kinase 1), TREM2 (Triggering Receptor Expressed On Myeloid Cells 2), and TRPM7 (Transient Receptor Potential Cation Channel Subfamily M Member 7).

The 15 gene variants, including VUS, detected in $13 \mathrm{Cau}-$ casian unrelated patients are listed in Table 1. All the variants were found in a heterozygous state.

Based on the American College of Medical Genetics and Genomics (ACMG) variant classification, one of the 15 detected gene variants was categorized as pathogenic, two as likely pathogenic and the remaining 12 as VUS.

Three genetic alterations found in our study had already been previously reported in ALS: TARDBP (c.1144G > A, p.Ala382Thr), a genetic alteration classified as pathogenic, that we found in an FALS patient and confirmed its presence in her affected brother (pedigree 1106). This variant is presented in population databases and was found in project MinE patients' dataset but not in controls. This variant has been reported in association with both familial and sporadic ALS [50].

Also, the likely pathogenic variant in $P R P H(\mathrm{c} .421 \mathrm{G}>\mathrm{T}$, p.Asp141Tyr) and the VUS found in FUS (c.1292C $>$ T, p.Pro431Leu) have already been described in ALS [51-53]. The $P R P H$ gene variant is found in population databases and in both ALS patients and controls from project MinE, and has conflicting interpretations of pathogenicity at ClinVar database. The FUS gene variant is found in population databases but only in ALS patients from project MinE (absent in controls) and it is classified as uncertain significance at ClinVar database.

Additionally, a novel variant c.1154G > T, (p.Trp385Leu) classified as likely pathogenic was found in a mutational hotspot for ALS-linked TARDBP variants (exon 6). Another variant in the same amino acid (p.Trp385Gly) has already been described in ALS [54] and most bioinformatic prediction tools indicate pathogenicity.

\section{Clinical characteristics of patients with pathogenic and likely pathogenic variants}

The clinical characteristics of patients harbouring gene variants are presented in Table 2.

Pathogenic and likely pathogenic variants were all found in FALS patients. In both pedigrees (1106 and 1363) with $T A R D B P$ variants, genetic anticipation was observed (i.e., appeared at an earlier age in the succeeding generation), although disease progression was not more severe, reflected in a similar rate of decay and long survival in both cases (Table 2). These patients from the second generation were not tested with NGS. To note, their disease onset characteristics were similar in sex, age, site, and UMN vs. LMN predominance. While \#1106 nephew developed FTD after long-disease duration, no cognitive alterations were noted in \#1363 son, who survived long time dependent of noninvasive ventilation (NIV). Among members of family 1106, clinical heterogeneity was observed.

The FALS patient with the PRPH (p.Asp141Tyr) is a female with bulbar-onset disease and concomitant FTD and LMN predominance.

\section{Variants uncertain significance (VUS)}

Variants classified as VUS were found in the following genes: NEFH, TRPM7, CHMP2B, ERBB4, TREM2, SPG11, and $C C N F$. Those found in TRPM7, CHMP $2 B, T B K 1$, and one of $N E F H$ (p.Lys741Gln) are novel variants; absent from population databases and from the current dataset of project MinE containing WGS data from 4366 ALS cases and 1832 controls. In silico predictions suggest pathogenicity for the TBK1 (c.682C > T, p.Arg228Cys), but are inconclusive regarding the CHMP $2 B$ (c.287 T $>$ C, p.Met96Thr), and suggest that the NEFH (c.2221A $>$ C, p.Lys741Gln) is non-pathogenic. The intronic variant (c.322-2A $>\mathrm{C})$ in TRPM7 gene is predicted to be splice altering (ada score: 1; rf score: 0.936$)$ and thus pathogenic. The rare variants found in $C C N F$ (p.Asn87Lys), both of ERBB4 (p.Phe414Leu) and (p.Ile910Val) and one of TREM2 (p.Glu151Lys), are not reported in ClinVar database. In silico prediction tools do not suggest them to be pathogenic, except for the $C C N F$. In fact, the $C C N F$ (p.Asn87Lys) is presented in patients from project MinE, but was not found in controls, though it is found in population databases with very low MAF. The TREM2 (p.Glu151Lys) is reported in population databases as well as in project MinE database. Both TREM2 variants (p.Glu151Lys) and (p.Arg47His) have been associated with the susceptibility to AD and most bioinformatic predictions suggest that they are likely benign. This variant is reported 


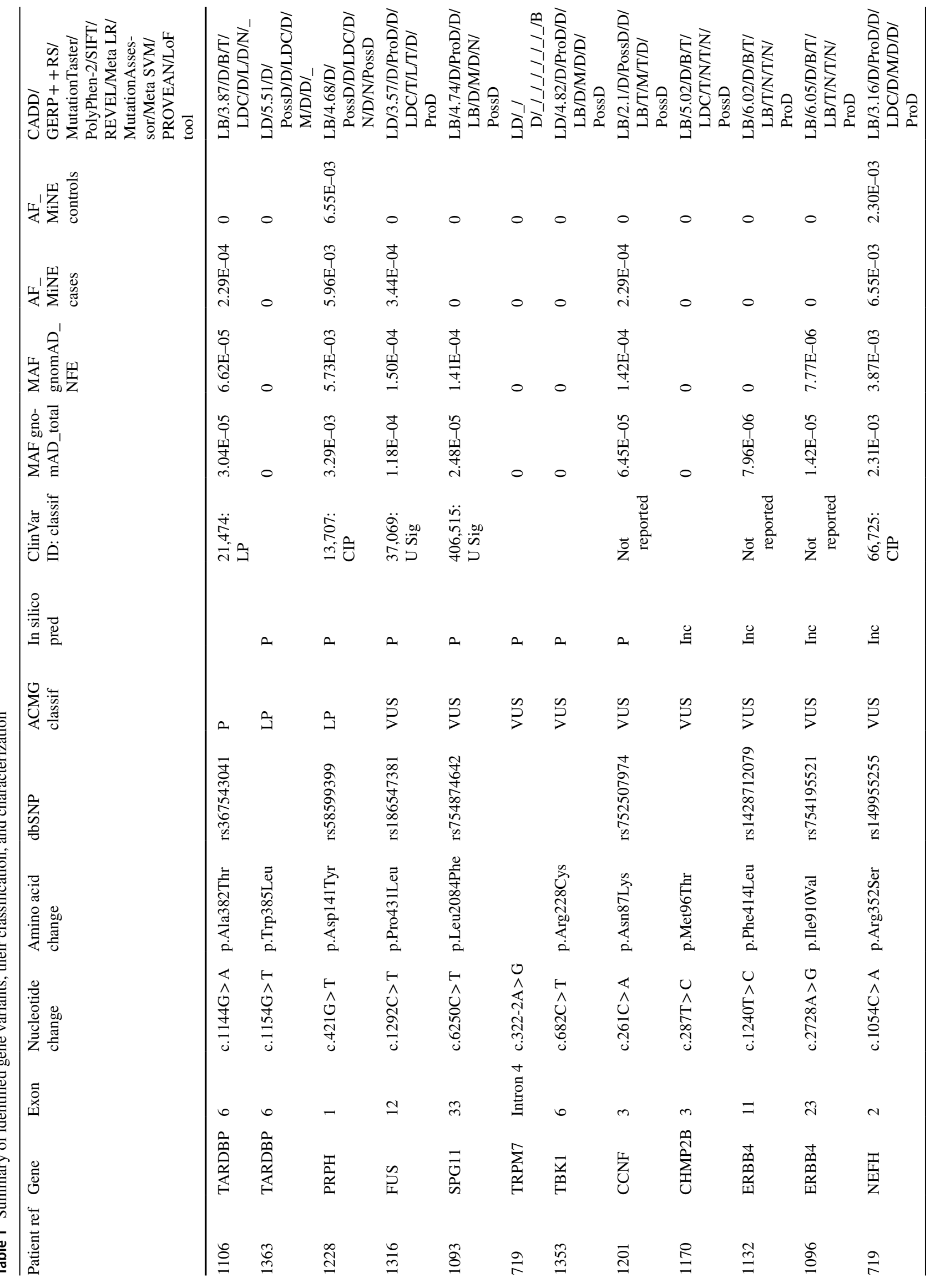




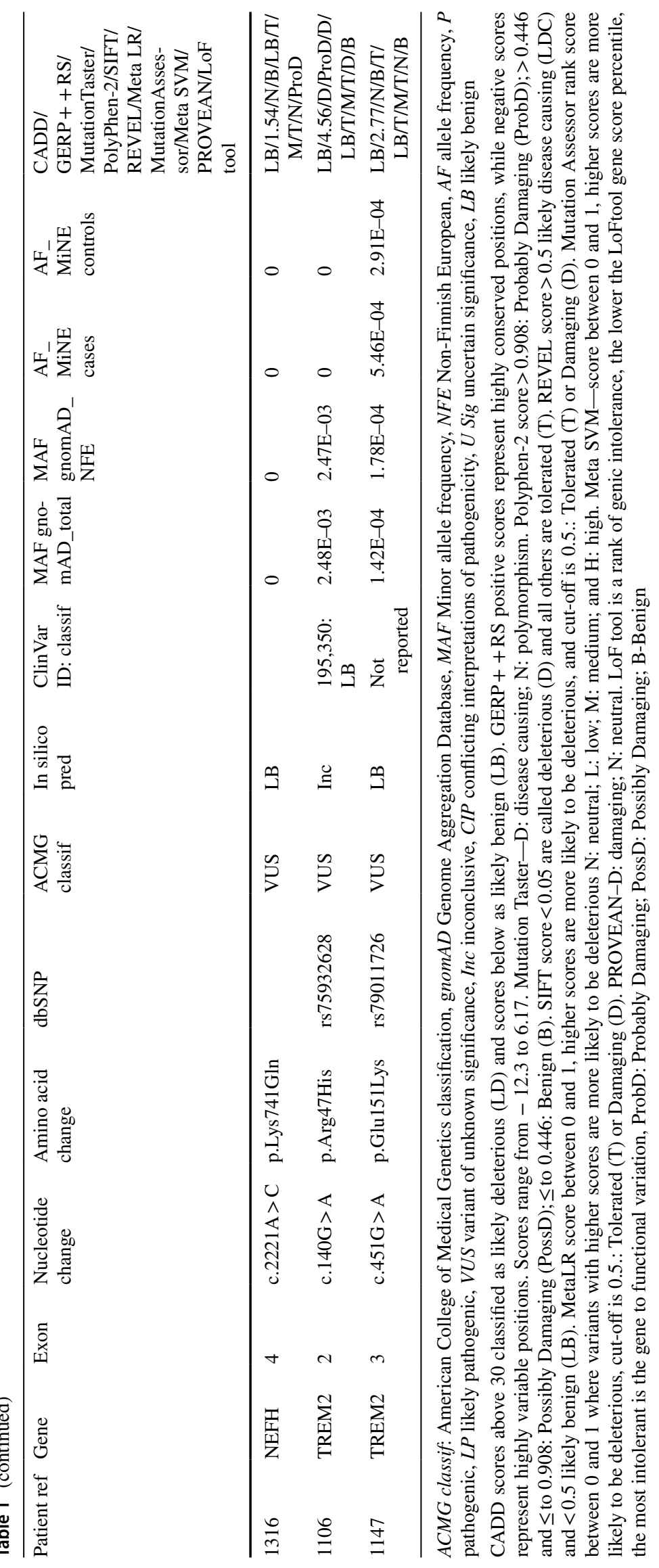




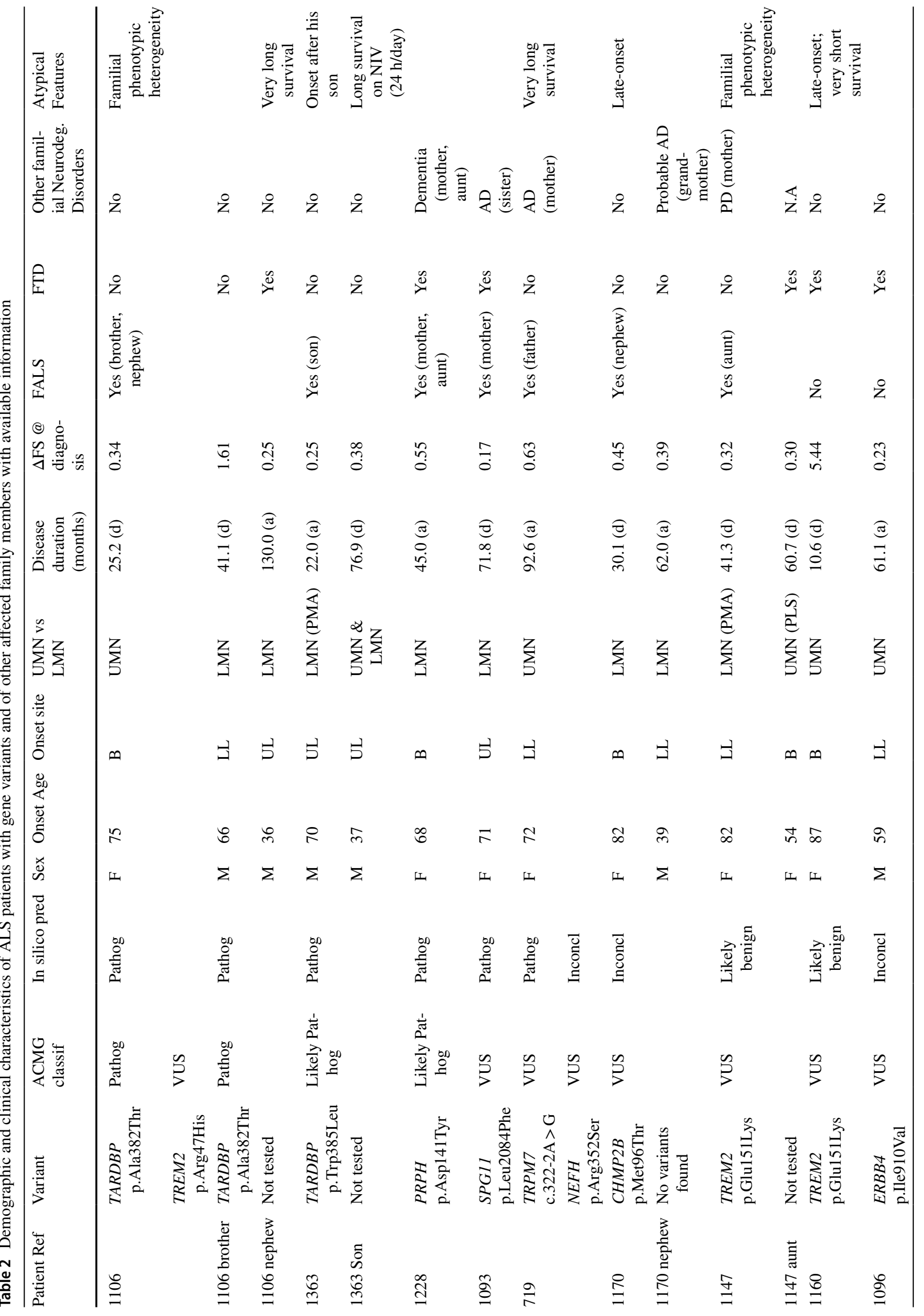




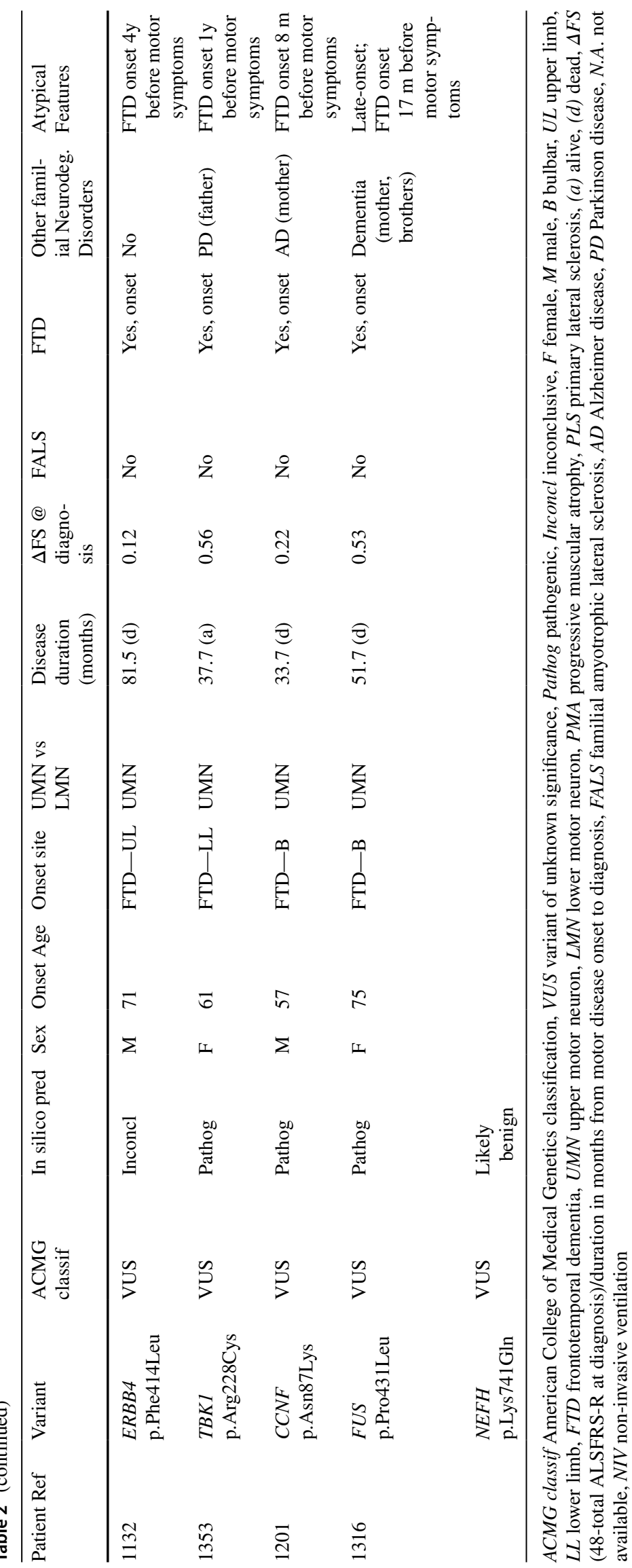


in population databases and in ALSdb with similar frequencies, but is not found in MinE database.

The NEFH (p.Arg352Ser) is presented in both population databases as well as in MinE dataset and has conflicting interpretations of pathogenicity.

The SPG11 (p.Leu2084Phe) is present in population databases, but was not found in MinE dataset, either in patients or controls, or in ALSdb. This variant is reported in ClinVar database associated with spastic paraplegia 11, autosomal recessive and classified with uncertain significance. A potential negative impact is suggested by bioinformatics tools; however, the available evidence is inconclusive. Moreover, the conditions related to SPG11 variants are inherited in an autosomal recessive pattern and we found it in heterozygosity.

The co-occurrence of multiple variants may be underestimated in our study, since those patients who were positive for $C 9$ orf 72 HRE were not further analysed with the NGS gene panel. Nevertheless, three patients harboured two rare variants in different genes $(8.8 \%, 3 / 34)$ : Patient \#1106, besides the pathogenic TARDBP (p.Ala382Thr) variant, also harboured the TREM2 (p.Arg47His) variant. This later VUS was not found in her affected brother. Unlike the other family members, patient \#1106 was the only with bulbar-onset and UMN predominance, the oldest at disease onset, and the one with the shortest survival (Table 2).

Patient \#719 has a novel splice acceptor variant in gene TRPM7 and another VUS in gene NEFH (p.Arg352Ser). This FALS patient has a very long survival and is still alive.

Patient \#1316, who had a VUS in FUS (p.Pro431Leu) and another in NEFH (p.Lys741Gln), had late FTD-onset disease that after 17 months progressed to the bulbar region.

In the majority of the analysed patients, no potential pathogenic variants were found $(59.4 \%, 19 / 32)$. Gene variants, including VUS, were found in 7 of the 17 FALS patients $(41.2 \%)$ and in 6 of the 15 SALS with concomitant FTD (40\%). Among those with FTD-onset, VUS were detected in $50 \%(4 / 8)$.

\section{Discussion}

In our study, we found 15 gene variants in 13 unrelated ALS patients negative for $C 9$ orf $72 \mathrm{HRE}$, and those variants were found in 11 genes: TARDBP, PRPH, SPG11, TRPM7, and $C H M P 2 B$ in FALS patients; ERBB4, FUS, TBK1, and $C C N F$ in SALS/FTD patients, and TREM 2 and NEFH in both SALS/FTD and FALS patients. Nevertheless, in 58.8\% of FALS and in $60 \%$ of SALS/FTD, no variants were identified in any of the analysed genes. This highlights that efforts, like those of project MinE [55], are still needed to identify additional genes influencing ALS/FTD risk.
Besides the major ALS genes (C9orf72, SOD1, TAR$D P B$, and $F U S$ ), recent extensive genetic screenings of ALS patients in European populations have identified variants in more rarely mutated genes in only about $4-5 \%$ of cases [56-59]. Moreover, those results reflect the ALS genetic diversity, even among European populations, since those variants are found in different genes and not even the major ALS genes are found or predominant in all cohorts, except the C9orf $72 \mathrm{HRE}$, confirming it as still the main genetic cause of ALS/FTD.

We found variants in FUS and TARDBP, but not in $S O D 1$, a gene that is rarely found mutated in Portuguese ALS patients [5].

Variants classified as pathogenic or likely pathogenic were only found in FALS patients in genes TARDBP and PRPH.

Both TARDBP variants were found in the mutational hotspot in exon 6 [50]. The p.Ala382Thr variant was initially described in two FALS patients of French origin [60]. Subsequently, large studies carried out on patients of Italian and French origin have identified this variant in FALS and SALS patients with or without FTD (reviewed in [50]). The incomplete penetrance of the disease in carriers at 70 years, calculated to be $74 \%$ in males and $42.5 \%$ in females [61], justifies why a 73-year-old female from family 1106 (sister and mother) is asymptomatic to date. We also found a new TARDBP p.Trp385Leu variant that is absent from population and ALS datasets; however, another variant in the same amino acid, c.1153T > G, p.Trp385Gly, was described in two affected brothers, one of which with very long-disease duration [54]. We found it in a PMA patient with disease onset long after his son who survived over 6 years, although for more than 2 years fully dependent on Non-Invasive Ventilation (NIV).

Disease anticipation was observed in both families and the patients from second generation (\#1106 nephew and \#1363 son) had similar disease onset characteristics. Despite that, considerable intrafamilial phenotypic differences in age, site of onset, and survival were observed in the families carrying the TARDBP variants, as previously reported [54], meaning that ALS manifestation is influenced by other genetic and environmental factors [62].

$P R P H$ p.Asp141Tyr was the first described variant related to ALS in this gene and was found in a homozygous sporadic patient with limb onset at 42 years old who died nearly 3 years after without cognitive changes [52]. It was also found in heterozygosity in 4/342 patients and 2/550 controls [51,63]. This variant does not abolish the ability of peripherin to assemble into filaments, but leads to the formation of aggregates, even in heterozygous state [52]. Although this functional evidence upholds that this could be a low penetrance variant involved in ALS susceptibility, the excess of its frequency in MinE controls and similar frequencies in 
ALS patients and in population databases argues against it, but there is also the possibility of having pre-symptomatic individuals in control populations. We found this variant in an FALS patient with bulbar-onset disease at 68 -years old and concomitant FTD that is still alive with almost 4 years of disease duration, different from the phenotype previously described [52] not confirming a genotype-phenotype relation for this variant.

In FALS patients, other variants of unknown significance were found in genes $S P G 11$, TRPM7, CHMP2B, TREM2, and NEFH.

The patient with the SPG11 p.Leu2084Phe missense variant also had concomitant FTD, a familial history of AD and upper limb onset with predominant LMN signs. Although SPG11 variants, mainly truncating mutations, have previously been associated with autosomal recessive juvenile ALS [64], several heterozygous missense variants in SPG11 have been reported in ALS, but attributed to the large size of the gene coding region and interpreted as unlikely deleterious [57, 59, 65-67].

The FALS patients with variants in $C H M P 2 B, T R P M 7$, $N E F H$, and TREM2 had no signs of FTD. However, variants in NEFH and TREM2 were also found in SALS/FTD patients.

The novel variant in $C H M P 2 B$ p.Met96Thr was found in an FALS patient with late bulbar-onset disease without FTD. A variant in the C-terminus of $C H M P 2 B$ was originally reported in a Danish pedigree with autosomal dominant FTD [68]. Other variants were later found in ALS and PMA in English and Dutch populations, $[69,70]$ and in one Indian SALS patient [71]. We did not find segregation of the variant with the disease in the other ALS patient of the family, what provides evidence against its pathogenicity.

The TRPM7 (c.322-2a > G) slice acceptor intronic variant is predicted to be pathogenic and is not reported in population or ALS databases. A missense variant in TRPM7 has been described to confer susceptibility to Guamanian ALS and Parkinsonian dementia [72]. The discrepancy in TRPM7 channel function and expression leads to various neuronal diseases such as AD and PD. Furthermore, it is a key factor in anoxic neuronal death and TRPM7 mutations may play a crucial role in neurotransmitter release in ALS [73]. We found the TRPM7 variant along with a VUS in NEFH p.Arg352Ser, in female patient with very long-disease survival (92.6 months, still alive) and whose father had ALS and mother had AD.

Neither NEFH variants were described in ALS; in fact, the p.Lys741Gln is a new variant not reported in population databases, but predicted to be likely benign by bioinformatic tools. Previously described clinical features observed in individuals with the NEFH (c.1054C > A, p.Arg352Ser) variant include: hypertonia, abnormality of coordination, morphological abnormality of the central nervous system, and neurophysiological changes (NCBI. ClinVar; [VCV000066725.3], https://www.ncbi.nlm.nih.gov/clinvar/ variation/VCV000066725.3 (accessed Feb. 20, 2020).

Further studies are needed to conclude about the pathogenicity of the detected VUS. Both were detected in patients who also had another variant. The NEFH p.Arg352Ser was detected along with the TRPM7 c.322-2A $>\mathrm{G}$ in an FALS patient with very long survival. The NEFH p.Lys741Gln was found in a patient with FTD-onset that also harboured a variant in FUS p.Pro431Gln.

TREM2 gene variants were reported to increase the risk of $\mathrm{AD}$ and other neurodegenerative diseases [74]. Recent meta-analysis studies have confirmed TREM2 p.Arg47His to be significantly associated with $\mathrm{AD}$ and PD risk in North Americans, but not PD in Europeans or ALS [74] and to be gene a risk factor for FTD in Caucasian populations [75]. The TREM2 p.Arg47His variant has also been associated with a neuroinflammatory processes, especially microglial activation $[76,77]$.

The TREM2 p.Glu151Lys was described before as risk factor for $\mathrm{AD}$ [78] and was found in a demented patient with LMN signs and Parkinsonism who was positive for the C9orf72 HRE [79]. We detected the TREM2 p.Glu151Lys variant in patients with late-onset disease (above the age of 80 ), one of which was a PMA patient with positive familial history, and the other was an SALS/FTD patient with UMN predominance and very short survival. The FALS patient with the TREM2 p.Arg47His variant also harboured the pathogenic TARDBP p.Ala382Thr, but in her brother, the TREM2 variant was not detected. The diverse neurodegenerative phenotypes associated with TREM2 variants indicate a role in neurodegeneration, although its risk effect in ALS remains elusive.

In the sporadic patients with FTD-onset, we found variants in genes FUS, TBK1, CCNF and ERBB4.

Since 2009, when the first FUS mutations were associated with ALS [80, 81], more than 50 variants have been identified in this gene $[50,82]$ with a frequency in European populations of about $2.8 \%$ in FALS and $0.3 \%$ in SALS [6]. Most pathogenic FUS variants are located in the C-terminal within, or completely deleting, the nuclear localization signal, thereby impairing nuclear import of FUS [83]. We found a variant at the zinc-finger motif, a rarely mutated site that has a predominant role in RNA recognition [84, 85]. This variant was first described in a patient with hereditary essential tremor-4 (ETM4; 614,782) whose lymphoblastoid cells showed lower expression of mutant FUS mRNA than did cells from patients with ALS due to FUS variants [86]. It was later found in an SALS Caucasian male patient with spinal-onset at the age of 60 [53], a quite different phenotype from our patient: a 75-year-old female with FTDonset that evolved to the bulbar region after 17 months. It is known that certain FUS genetic variants do result in very 
different phenotypes $[83,87]$ emphasizing the influence of other factors in disease manifestation which, in this case, could be related with the presence of the $N E F H$ variant p.(Lys741Gln), even if it is predicted to be likely benign. Also, the lack of significant numbers of non-nuclear localization signal variants preclude the description of their phenotypes properly [83]. FUS mutation carriers usually manifest earlier symptom onset, a higher rate of bulbar-onset, and shorter disease duration. Nonetheless, FTD has rarely been reported among ALS patients with FUS variants [87-89].

Whole-exome sequencing and a gene-based rare variant analysis identified TBK1 as an ALS gene [37, 90]. In ALS-FTD patients, most reported pathogenic $T B K 1$ variants are loss-of-function (LoF) mutations [90-92] and functional TBK1 missense variants have been proposed to be considered high risk rather than causal variants [92]. However, in vitro tests have demonstrated that at least some missense variants have functional deficits and, therefore, may be disease-causing [90, 93]. Furthermore, a recent meta-analysis has shown that LoF mutations are less frequent than missense variants in ALS-FTD and both are associated with an increased risk for ALS-FTD spectrum. However, while $T B K 1$ LoF mutations were associated with a significant increased risk, missense variants of $T B K 1$ were only related to a moderately increased susceptibility [94]. We found a missense variant predicted to be pathogenic by in silico tools in a patient with FTD-onset that progressed to lower limbs 1 year after and whose father had PD.

$C C N F$ was found as ALS causative gene in a genomewide linkage analysis in large ALS-FTD kindred [95]. The analysis of a replication cohort suggested its role in both FALS and SALS pathogenesis through abnormal ubiquitination and accumulation of ubiquitinated proteins, including TDP-43 [95]. We found a rare variant, predicted to be pathogenic, in an SALS patient with FTD-onset disease that progressed to the bulbar region after 8 months and whose mother had AD/dementia.

Rare genetic variants in the ERBB 4, a member of the epidermal growth factor subfamily of receptor tyrosine kinases, were detected in different pedigrees of FALS patients by whole-genome sequencing and parametric linkage analysis [96]. Clinical presentations of those individuals were characterized by the involvement of both UMN and LMN, a lack of obvious cognitive dysfunction, and relatively slow progression. ERBB 4 variants were later found in patients with ALS with concomitant FTD $[23,97]$ some evidencing incomplete penetrance [97]. Both our patients with ERBB4 variants had UMN predominance and concomitant FTD; one of them presented with FTD 4 years before upper limb symptoms. No familial history of ALS or any other neurodegenerative disease was uncovered.

Although both found variants are rare and in silico predictions are inconclusive, p.Ile910Val is located within the tyrosine kinase domain and p.Phe414Leu within a receptor domain, suggesting that these variants might impact receptor ligand interaction and kinase activity, key functions of ErbB4.

In summary, regarding the VUS found in FUS, SPG11, TRPM7, TBK1, CCNF, ERBB4, NEFH, and TREM2, no definite conclusion regarding its pathogenicity can be drawn and further functional, genetic, and clinical studies in large independent cohorts, which are required to fully understand and establish their contribution for ALS.

In agreement with several studies that have shown an oligogenic basis of ALS [12, 53, 57, 98-100] as the number of patients with multiple ALS-associated variants is higher than what can be expected by chance, based on the individual mutation frequencies of the respective genes, we also found three patients carrying two potential disease-causing variants. Thus, certain variants alone may not cause disease and the simultaneous analysis of disease genes is highly important [59].

For those variants that had already been described in ALS, we observed some consistency of phenotypes with those previously reported, supporting possible genotype-phenotype relation, but mainly, we found distinctive characteristics highlighting the influence of other genetic and non-genetic factors in ALS manifestation.

A recent population-based parent-offspring heritability study showed that inherited and non-inherited factors contribute approximately equally toward ALS, and that even in a population devoid of known gene mutations, genetic factors account for almost $40 \%$ of the risk [14].

All the above emphasize the need to continue and enhance the efforts to unravel the genetic architecture of ALS-FTD mindful that only a better knowledge of the disease will give us the basis to fight it.

Author contributions Conceptualization, MG and MdC; methodology, ACPL and JT; software, MG and JT; validation, DA; formal analysis, MG, AMC, and RR; data curation, $\mathrm{MG}, \mathrm{AMC}, \mathrm{RR}$, and DA; writingoriginal draft preparation, MG; writing - review and editing, MG and MdC; supervision, MdC.

Funding This project was supported by FTC-Fundação para a Ciência e Tecnologia - through project "Comprehensive evaluation of circulating MicroRNA as diagnostic and prognostic biomarkers in Amyotrophic Lateral Sclerosis"-PTDC/MEC-NEU/31195/2017.

Availability of data and material Further data are available upon request.

\section{Compliance with ethical standards}

Conflicts of interest The authors declare that they have no conflict of interest.

Ethics approval This project was approved by the Local Ethics Committee (ID number 215/15). The study conformed to the standards 
defined in the latest revision of the Declaration of Helsinki. All patients and controls signed a written informed consent. Databases were properly treated for privacy.

Consent to participate All listed authors have approved the manuscript before submission, including the names and order of authors.

Consent for publication All listed authors have approved the version to be published.

\section{References}

1. van Es MA, Hardiman O, Chio A et al (2017) Amyotrophic lateral sclerosis. Lancet 390:2084-2098. https://doi.org/10.1016/ S0140-6736(17)31287-4

2. Lomen-Hoerth C, Anderson T, Miller B (2002) The overlap of amyotrophic lateral sclerosis and frontotemporal dementia. Neurology 59:1077-1079. https://doi.org/10.1212/wnl.59.7.1077

3. Ryan M, Heverin M, Doherty MA et al (2018) Determining the incidence of familiality in ALS. Neurol Genet. https://doi. org/10.1212/NXG.0000000000000239

4. Byrne S, Heverin M, Elamin M et al (2013) Aggregation of neurologic and neuropsychiatric disease in amyotrophic lateral sclerosis kindreds: a population-based case-control cohort study of familial and sporadic amyotrophic lateral sclerosis. Ann Neurol 74:699-708. https://doi.org/10.1002/ana.23969

5. Gromicho M, Pinto S, Gisca E et al (2018) Frequency of C9orf72 hexanucleotide repeat expansion and SOD1 mutations in Portuguese patients with amyotrophic lateral sclerosis. Neurobiol Aging 70:325.e7-325.e15. https://doi.org/10.1016/j.neurobiola ging.2018.05.009

6. Zou Z-Y, Zhou Z-R, Che C-H et al (2017) Genetic epidemiology of amyotrophic lateral sclerosis: a systematic review and metaanalysis. J Neurol Neurosurg Psychiatry. https://doi.org/10.1136/ jnnp-2016-315018

7. van der Zee J, Gijselinck I, Dillen L et al (2013) A Pan-European Study of the C9orf72 Repeat Associated with FTLD: Geographic Prevalence, Genomic Instability, and Intermediate Repeats. Hum Mutat 34:363-373. https://doi.org/10.1002/humu.22244

8. Cruts M, Gijselinck I, Van Langenhove T et al (2013) Current insights into the $\mathrm{C} 9$ orf72 repeat expansion diseases of the FTLD/ALS spectrum. Trends Neurosci 36:450-459. https://doi. org/10.1016/j.tins.2013.04.010

9. Majounie E, Renton AE, Mok K et al (2012) Frequency of the C9orf72 hexanucleotide repeat expansion in patients with amyotrophic lateral sclerosis and frontotemporal dementia: a cross-sectional study. Lancet Neurol 11:323-330. https://doi. org/10.1016/S1474-4422(12)70043-1

10. Renton AE, Majounie E, Waite A et al (2011) A hexanucleotide repeat expansion in C9ORF72 is the cause of chromosome 9p21linked ALS-FTD. Neuron 72:257-268. https://doi.org/10.1016/j. neuron.2011.09.010

11. DeJesus-Hernandez M, Mackenzie IR, Boeve BF et al (2011) Expanded GGGGCC hexanucleotide repeat in noncoding region of C9ORF72 causes chromosome 9p-linked FTD and ALS. Neuron 72:245-256. https://doi.org/10.1016/j.neuron.2011.09.011

12. Lattante S, Ciura S, Rouleau GA, Kabashi E (2015) Defining the genetic connection linking amyotrophic lateral sclerosis (ALS) with frontotemporal dementia (FTD). Trends Genet 31:263-273. https://doi.org/10.1016/j.tig.2015.03.005

13. Gao F, Almeida S, Lopez-Gonzalez R (2017) Dysregulated molecular pathways in amyotrophic lateral sclerosis-frontotemporal dementia spectrum disorder. EMBO J 36:2931-2950. https://doi.org/10.15252/embj.201797568

14. Ryan M, Heverin M, McLaughlin RL, Hardiman O (2019) Lifetime risk and heritability of amyotrophic lateral sclerosis. JAMA Neurol 76:1367. https://doi.org/10.1001/jamaneurol .2019 .2044

15. Mathis S, Goizet C, Soulages A et al (2019) Genetics of amyotrophic lateral sclerosis: a review. J Neurol Sci 399:217-226. https://doi.org/10.1016/j.jns.2019.02.030

16. Chia R, Chiò A, Traynor BJ (2018) Novel genes associated with amyotrophic lateral sclerosis: diagnostic and clinical implications. Lancet Neurol 17:94-102. https://doi.org/10.1016/S1474 $-4422(17) 30401-5$

17. Farhan SMK, Howrigan DP, Abbott LE et al (2019) Exome sequencing in amyotrophic lateral sclerosis implicates a novel gene, DNAJC7, encoding a heat-shock protein. Nat Neurosci 22:1966-1974. https://doi.org/10.1038/s41593-019-0530-0

18. Nguyen HP, Van Broeckhoven C, van der Zee J (2018) ALS genes in the genomic era and their implications for FTD. Trends Genet 34:404-423. https://doi.org/10.1016/j.tig.2018.03.001

19. Al-Chalabi A, Calvo A, Chio A et al (2014) Analysis of amyotrophic lateral sclerosis as a multistep process: a populationbased modelling study. Lancet Neurol 13:1108-1113. https:// doi.org/10.1016/S1474-4422(14)70219-4

20. Vucic S, Higashihara M, Sobue G et al (2020) ALS is a multistep process in South Korean, Japanese, and Australian patients. Neurology. https://doi.org/10.1212/WNL.000000000000901 5.10.1212/WNL.0000000000009015

21. Chiò A, Mazzini L, D'Alfonso S et al (2018) The multistep hypothesis of ALS revisited. Neurology 91:e635-e642. https:// doi.org/10.1212/WNL.0000000000005996

22. Chiò A, Calvo A, Mazzini L et al (2012) Extensive genetics of ALS: a population-based study in Italy extensive genetics of ALS a population-based study in Italy. Neurology. https://doi. org/10.1212/WNL.0b013e3182735d36

23. Dols-Icardo O, García-Redondo A, Rojas-García R et al (2018) Analysis of known amyotrophic lateral sclerosis and frontotemporal dementia genes reveals a substantial genetic burden in patients manifesting both diseases not carrying the C9orf72 expansion mutation. J Neurol Neurosurg Psychiatry 89:162-168. https://doi.org/10.1136/jnnp-2017-316820

24. Turner MR, Al-Chalabi A, Chio A et al (2017) Genetic screening in sporadic ALS and FTD. J Neurol Neurosurg Psychiatry. https ://doi.org/10.1136/jnnp-2017-315995

25. Brooks BR, Miller RG, Swash M, Munsat TL (2000) El Escorial revisited: Revised criteria for the diagnosis of amyotrophic lateral sclerosis. Amyotroph Lateral Scler Other Mot Neuron Disord 1:293-299. https://doi.org/10.1080/146608200300079536

26. de Carvalho M, Dengler R, Eisen A et al (2008) Electrodiagnostic criteria for diagnosis of ALS. Clin Neurophysiol 119:497503. https://doi.org/10.1016/j.clinph.2007.09.143

27. De Carvalho M, Ryczkowski A, Andersen P et al (2017) International survey of ALS experts about critical questions for assessing patients with ALS. Amyotroph Lateral Scler Front Degener. https://doi.org/10.1080/21678421.2017.1349150

28. Byrne S, Bede P, Elamin M et al (2011) Proposed criteria for familial amyotrophic lateral sclerosis. Amyotroph Lateral Scler 12:157-159. https://doi.org/10.3109/17482968.2010.545420

29. Rascovsky K, Hodges JR, Knopman D et al (2011) Sensitivity of revised diagnostic criteria for the behavioural variant of frontotemporal dementia. Brain 134:2456-2477. https://doi. org/10.1093/brain/awr179

30. Cedarbaum JM, Stambler N, Malta E et al (1999) The ALSFRS$\mathrm{R}$ : a revised ALS functional rating scale that incorporates assessments of respiratory function. J Neurol Sci 169:13-21. https:// doi.org/10.1016/S0022-510X(99)00210-5 
31. Akimoto C, Volk AE, van Blitterswijk M et al (2014) A blinded international study on the reliability of genetic testing for GGG GCC-repeat expansions in C9orf72 reveals marked differences in results among 14 laboratories. J Med Genet 51:419-424. https ://doi.org/10.1136/jmedgenet-2014-102360

32. McKenna A, Hanna M, Banks E et al (2010) The Genome Analysis Toolkit: a MapReduce framework for analyzing next-generation DNA sequencing data. Genome Res 20:1297-1303. https:// doi.org/10.1101/gr.107524.110

33. Garrison E, Marth G (2012) Haplotype-based variant detection from short-read sequencing. arXiv pre-print Serv

34. Li H, Handsaker B, Wysoker A et al (2009) The sequence alignment/map format and SAMtools. Bioinformatics 25:2078-2079. https://doi.org/10.1093/bioinformatics/btp352

35. Whiffin N, Minikel E, Walsh R et al (2017) Using high-resolution variant frequencies to empower clinical genome interpretation. Genet Med 19:1151-1158. https://doi.org/10.1038/gim.2017.26

36. van der Spek RAA, van Rheenen W, Pulit SL et al (2019) The project MinE databrowser: bringing large-scale whole-genome sequencing in ALS to researchers and the public. Amyotroph Lateral Scler Front Degener 20:432-440. https://doi. org/10.1080/21678421.2019.1606244

37. Cirulli ET, Lasseigne BN, Petrovski S et al (2015) Exome sequencing in amyotrophic lateral sclerosis identifies risk genes and pathways. Science 347:1436-1441. https://doi.org/10.1126/ science.aaa3650

38. Richards S, Aziz N, Bale S et al (2015) Standards and guidelines for the interpretation of sequence variants: a joint consensus recommendation of the American College of Medical Genetics and Genomics and the Association for Molecular Pathology. Genet Med 17:405-424. https://doi.org/10.1038/gim.2015.30

39. Schwarz JM, Cooper DN, Schuelke M, Seelow D (2014) MutationTaster2: mutation prediction for the deep-sequencing age. Nat Methods 11:361-362. https://doi.org/10.1038/nmeth.2890

40. Adzhubei IA, Schmidt S, Peshkin L et al (2010) A method and server for predicting damaging missense mutations. Nat Methods $7: 248$

41. Sim N-L, Kumar P, Hu J et al (2012) SIFT web server: predicting effects of amino acid substitutions on proteins. Nucleic Acids Res 40:W452-W457

42. Ioannidis NM, Rothstein JH, Pejaver V et al (2016) REVEL: an ensemble method for predicting the pathogenicity of rare missense variants. Am J Hum Genet 99:877-885. https://doi. org/10.1016/j.ajhg.2016.08.016

43. Reva B, Antipin Y, Sander C (2007) Determinants of protein function revealed by combinatorial entropy optimization. Genome Biol 8:R232. https://doi.org/10.1186/gb-2007-8-11-r232

44. Dong C, Wei P, Jian X et al (2015) Comparison and integration of deleteriousness prediction methods for nonsynonymous SNVs in whole exome sequencing studies. Hum Mol Genet 24:21252137. https://doi.org/10.1093/hmg/ddu733

45. Davydov EV, Goode DL, Sirota M et al (2010) Identifying a high fraction of the human genome to be under selective constraint using GERP++. PLOS Comput Biol 6:e1001025

46. McLaren W, Gil L, Hunt SE et al (2016) The ensembl variant effect predictor. Genome Biol 17:122. https://doi.org/10.1186/ s13059-016-0974-4

47. Liu X, Wu C, Li C, Boerwinkle E (2016) dbNSFP v3.0: a one-stop database of functional predictions and annotations for human nonsynonymous and splice-site SNVs. Hum Mutat 37:235-241. https://doi.org/10.1002/humu.22932

48. Fadista J, Oskolkov N, Hansson O, Groop L (2017) LoFtool: a gene intolerance score based on loss-of-function variants in 60706 individuals. Bioinformatics 33:471-474. https://doi. org/10.1093/bioinformatics/btv602
49. Jian X, Boerwinkle E, Liu X (2014) In silico prediction of splicealtering single nucleotide variants in the human genome. Nucleic Acids Res 42:13534-13544. https://doi.org/10.1093/nar/gku1206

50. Lattante S, Rouleau GA, Kabashi E (2013) TARDBP and FUS mutations associated with amyotrophic lateral sclerosis: summary and update. Hum Mutat 34:812-826. https://doi. org/10.1002/humu.22319

51. Corrado L, Carlomagno Y, Falasco L et al (2011) A novel peripherin gene (PRPH) mutation identified in one sporadic amyotrophic lateral sclerosis patient. Neurobiol Aging 32:552. e1-552.e6. https://doi.org/10.1016/j.neurobiolaging.2010.02.011

52. Leung CL, He CZ, Kaufmann P et al (2004) A pathogenic peripherin gene mutation in a patient with amyotrophic lateral sclerosis. Brain Pathol 14:290-296. https://doi. org/10.1111/j.1750-3639.2004.tb00066.x

53. Cady J, Allred P, Bali T et al (2015) Amyotrophic lateral sclerosis onset is influenced by the burden of rare variants in known amyotrophic lateral sclerosis genes. Ann Neurol 77:100-113. https:// doi.org/10.1002/ana.24306

54. Millecamps S, Salachas F, Cazeneuve C et al (2010) SOD1, ANG, VAPB, TARDBP, and FUS mutations in familial amyotrophic lateral sclerosis: Genotype-phenotype correlations. J Med Genet 47:554-560. https://doi.org/10.1136/jmg.2010.077180

55. Project MinE ALS Sequencing Consortium (2018) Project MinE: study design and pilot analyses of a large-scale wholegenome sequencing study in amyotrophic lateral sclerosis. Eur J Hum Genet 26:1537-1546. https://doi.org/10.1038/s4143 1-018-0177-4

56. Pensato, Magri, Bella et al (2020) Sorting rare ALS genetic variants by targeted re-sequencing panel in Italian patients: OPTN, $\mathrm{VCP}$, and SQSTM1 variants account for $3 \%$ of rare genetic forms. J Clin Med 9:412. https://doi.org/10.3390/jcm9020412

57. Tripolszki K, Gampawar P, Schmidt H et al (2019) Comprehensive genetic analysis of a hungarian amyotrophic lateral sclerosis cohort. Front Genet 10:732. https://doi.org/10.3389/fgene .2019.00732

58. Müller K, Brenner D, Weydt P et al (2018) Comprehensive analysis of the mutation spectrum in 301 German ALS families. J Neurol Neurosurg Psychiatry 89:817-827. https://doi.org/10.1136/ jnnp-2017-317611

59. Kenna KP, McLaughlin RL, Byrne S et al (2013) Delineating the genetic heterogeneity of ALS using targeted high-throughput sequencing. J Med Genet 50:776-783. https://doi.org/10.1136/ jmedgenet-2013-101795

60. Kabashi E, Valdmanis PN, Dion P et al (2008) TARDBP mutations in individuals with sporadic and familial amyotrophic lateral sclerosis. Nat Genet 40:572-574. https://doi.org/10.1038/ ng. 132

61. Orrù $S$, Manolakos E, Orrù $N$ et al (2012) High frequency of the TARDBP p.Ala382Thr mutation in Sardinian patients with amyotrophic lateral sclerosis. Clin Genet 81:172-178. https://doi. org/10.1111/j.1399-0004.2011.01668.x

62. Borghero G, Pugliatti M, Marrosu F et al (2014) Genetic architecture of ALS in Sardinia. Neurobiol Aging 35:2882.e7-2882. e12. https://doi.org/10.1016/j.neurobiolaging.2014.07.012

63. Gros-Louis F, Larivière R, Gowing G et al (2004) A frameshift deletion in peripherin gene associated with amyotrophic lateral sclerosis. J Biol Chem 279:45951-45956. https://doi. org/10.1074/jbc.M408139200

64. Orlacchio A, Babalini C, Borreca A et al (2010) SPATACSIN mutations cause autosomal recessive juvenile amyotrophic lateral sclerosis. Brain 133:591-598. https://doi.org/10.1093/brain lawp325

65. Couthouis J, Raphael AR, Daneshjou R, Gitler AD (2014) Targeted exon capture and sequencing in sporadic amyotrophic 
lateral sclerosis. PLoS Genet 10:e1004704. https://doi. org/10.1371/journal.pgen.1004704

66. Nakamura R, Sone J, Atsuta N et al (2016) Next-generation sequencing of 28 ALS-related genes in a Japanese ALS cohort. Neurobiol Aging 39:219.e1-219.e8. https://doi.org/10.1016/j. neurobiolaging.2015.11.030

67. Nishiyama A, Niihori T, Warita $\mathrm{H}$ et al (2017) Comprehensive targeted next-generation sequencing in Japanese familial amyotrophic lateral sclerosis. Neurobiol Aging 53:194.e1-194.e8. https://doi.org/10.1016/j.neurobiolaging.2017.01.004

68. Parkinson N, Ince PG, Smith MO et al (2006) ALS phenotypes with mutations in CHMP2B (charged multivesicular body protein 2B). Neurology 67:1074-1077. https://doi.org/10.1212/01. wnl.0000231510.89311.8b

69. Cox LE, Ferraiuolo L, Goodall EF et al (2010) Mutations in CHMP2B in lower motor neuron predominant amyotrophic lateral sclerosis (ALS). PLoS ONE 5:e9872. https://doi. org/10.1371/journal.pone.0009872

70. van Blitterswijk M, Vlam L, van Es MA et al (2012) Genetic overlap between apparently sporadic motor neuron diseases. PLoS ONE 7:e48983. https://doi.org/10.1371/journ al.pone. 0048983

71. Narain P, Pandey A, Gupta S et al (2018) Targeted next-generation sequencing reveals novel and rare variants in Indian patients with amyotrophic lateral sclerosis. Neurobiol Aging 71:265.e9-265.e14. https://doi.org/10.1016/j.neurobiola ging.2018.05.012

72. Hermosura MC, Nayakanti H, Dorovkov MV et al (2005) A TRPM7 variant shows altered sensitivity to magnesium that may contribute to the pathogenesis of two Guamanian neurodegenerative disorders. Proc Natl Acad Sci USA 102:11510-11515. https ://doi.org/10.1073/pnas.0505149102

73. Sun Y, Sukumaran P, Schaar A, Singh BB (2015) TRPM7 and its role in neurodegenerative diseases. Channels 9:253-261. https:// doi.org/10.1080/19336950.2015.1075675

74. Zhou S-L, Tan C-C, Hou X-H et al (2019) TREM2 variants and neurodegenerative diseases: a systematic review and meta-analysis. J Alzheimers Dis 68:1171-1184. https://doi.org/10.3233/ JAD-181038

75. Su W-H, Shi Z-H, Liu S-L et al (2018) The rs75932628 and rs2234253 polymorphisms of the TREM2 gene were associated with susceptibility to frontotemporal lobar degeneration in Caucasian populations. Ann Hum Genet 82:177-185. https://doi. org/10.1111/ahg.12241

76. Cady J, Koval ED, Benitez BA et al (2014) TREM2 variant p. $\mathrm{R} 47 \mathrm{H}$ as a risk factor for sporadic amyotrophic lateral sclerosis. JAMA Neurol 71:449-453. https://doi.org/10.1001/jamaneurol .2013 .6237

77. Ogaki K, Heckman MG, Koga S et al (2018) Association study between multiple system atrophy and TREM 2 p. R47H. Neurol Genet 4:e257-e257. https://doi.org/10.1212/NXG.0000000000 000257

78. Jin SC, Benitez BA, Karch CM et al (2014) Coding variants in TREM2 increase risk for Alzheimer's disease. Hum Mol Genet 23:5838-5846. https://doi.org/10.1093/hmg/ddu277

79. Giannoccaro MP, Bartoletti-Stella A, Piras S et al (2017) Multiple variants in families with amyotrophic lateral sclerosis and frontotemporal dementia related to C9orf72 repeat expansion: further observations on their oligogenic nature. J Neurol 264:1426-1433. https://doi.org/10.1007/s00415-017-8540-x

80. Kwiatkowski TJ, Bosco DA, Leclerc AL et al (2009) Mutations in the FUS/TLS gene on chromosome 16 cause familial amyotrophic lateral sclerosis. Science 323:1205-1208. https://doi. org/10.1126/science.1166066

81. Vance C, Rogelj B, Hortobagyi T et al (2009) Mutations in FUS, an RNA processing protein, cause familial amyotrophic lateral sclerosis type 6. Science 323:1208-1211. https://doi. org/10.1126/science.1165942

82. Mejzini R, Flynn LL, Pitout IL et al (2019) ALS genetics, mechanisms, and therapeutics: where are we now? Front Neurosci 13:1-27. https://doi.org/10.3389/fnins.2019.01310

83. Naumann M, Peikert K, Günther R et al (2019) Phenotypes and malignancy risk of different FUS mutations in genetic amyotrophic lateral sclerosis. Ann Clin Transl Neurol 6:2384-2394. https://doi.org/10.1002/acn3.50930

84. Iko Y, Kodama TS, Kasai N et al (2004) Domain architectures and characterization of an RNA-binding protein, TLS. J Biol Chem 279:44834-44840. https://doi.org/10.1074/jbc.M4085 52200

85. Shang Y, Huang EJ (2016) Mechanisms of FUS mutations in familial amyotrophic lateral sclerosis. Brain Res 1647:65-78. https://doi.org/10.1016/j.brainres.2016.03.036

86. Merner ND, Girard SL, Catoire H et al (2012) Exome sequencing identifies FUS mutations as a cause of essential tremor. Am J Hum Genet 91:313-319. https://doi.org/10.1016/j. ajhg.2012.07.002

87. Yan J, Deng HX, Siddique N et al (2010) Frameshift and novel mutations in FUS in familial amyotrophic lateral sclerosis and ALS/dementia. Neurology 75:807-814. https://doi. org/10.1212/WNL.0b013e3181f07e0c

88. Akiyama T, Warita H, Kato M et al (2016) Genotype-phenotype relationships in familial amyotrophic lateral sclerosis with FUS/TLS mutations in Japan. Muscle Nerve 54:398-404. https ://doi.org/10.1002/mus.25061

89. Van Langenhove T, van der Zee J, Sleegers K et al (2010) Genetic contribution of FUS to frontotemporal lobar degeneration. Neurology 74:366-371. https://doi.org/10.1212/ WNL.0b013e3181ccc732

90. Freischmidt A, Wieland T, Richter B et al (2015) Haploinsufficiency of TBK1 causes familial ALS and fronto-temporal dementia. Nat Neurosci 18:631-636. https://doi.org/10.1038/ nn. 4000

91. Costa MR, Gromicho M, Pronto-Laborinho AC et al (2019) Novel TBK1 LoF variant in a family with upper motor neuron predominant motor neuron disease. J Neurol Sci 403:117-118. https://doi.org/10.1016/j.jns.2019.06.029

92. van der Zee J, Gijselinck I, Van Mossevelde S et al (2017) TBK1 mutation spectrum in an extended European patient cohort with frontotemporal dementia and amyotrophic lateral sclerosis. Hum Mutat 38:297-309. https://doi.org/10.1002/ humu.23161

93. Freischmidt A, Müller K, Ludolph AC et al (2017) Association of mutations in TBK1 with sporadic and familial amyotrophic lateral sclerosis and frontotemporal dementia. JAMA Neurol 74:110-113. https://doi.org/10.1001/jamaneurol.2016.3712

94. Cui R, Tuo M, Li P, Zhou C (2018) Association between TBK1 mutations and risk of amyotrophic lateral sclerosis/frontotemporal dementia spectrum: a meta-analysis. Neurol Sci 39:811-820. https://doi.org/10.1007/s10072-018-3246-0

95. Williams KL, Topp S, Yang S et al (2016) CCNF mutations in amyotrophic lateral sclerosis and frontotemporal dementia. Nat Commun 7:11253. https://doi.org/10.1038/ncomms11253

96. Takahashi Y, Fukuda Y, Yoshimura J et al (2013) ERBB4 mutations that disrupt the neuregulin-ErbB4 pathway cause amyotrophic lateral sclerosis type 19. Am J Hum Genet 93:900-905. https://doi.org/10.1016/j.ajhg.2013.09.008

97. Sun L, Cheng B, Zhou Y et al (2020) ErbB4 mutation that decreased NRG1-ErbB4 signaling involved in the pathogenesis of amyotrophic lateral sclerosis/frontotemporal dementia. J Alzheimer's Dis. https://doi.org/10.3233/JAD-191230

98. Van Blitterswijk M, Van Es MA, Hennekam EAM et al (2012) Evidence for an oligogenic basis of amyotrophic lateral sclerosis. 
Hum Mol Genet 21:3776-3784. https://doi.org/10.1093/hmg/ dds 199

99. Lattante S, Doronzio PN, Marangi G et al (2019) Coexistence of variants in TBK1 and in other ALS-related genes elucidates an oligogenic model of pathogenesis in sporadic ALS. Neurobiol Aging 84:239.e9-239.e14. https://doi.org/10.1016/j.neurobiola ging.2019.03.010
100. Zhang H, Cai W, Chen S et al (2018) Screening for possible oligogenic pathogenesis in Chinese sporadic ALS patients. Amyotroph Lateral Scler Frontotemporal Degener 19:419-425. https ://doi.org/10.1080/21678421.2018.1432659 